\title{
Aptitud territorial para cultivo de tilapia (Oreochromis niloticus) con tecnología biofloc en el estado de Durango, México
}

\section{Land suitability for growing tilapia (Oreochromis niloticus) with biofloc technology in the state of Durango, México}

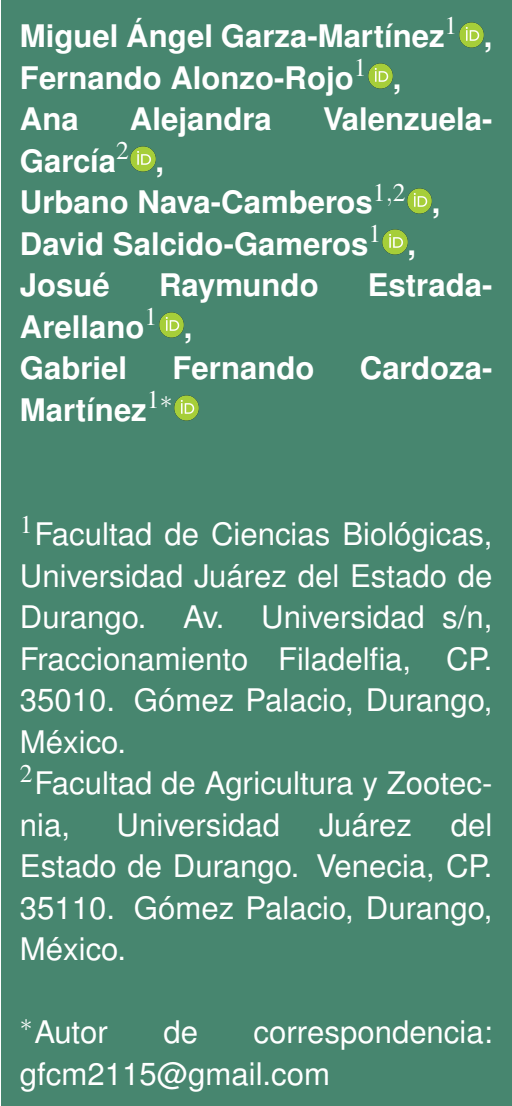

Artículo científico

Recibido: 07 de junio 2021

Aceptado: 20 de Agosto 2021

Como citar: Garza-Martínez MA Alonzo-Rojo F, Valenzuela-García AA, Nava-Camberos U, SalcidoGameros D, Estrada-Arellano JR, Cardoza-Martínez GF (2021) Aptitud territorial para cultivo de tilapia (Oreochromis niloticus) con tecnología biofloc en el estado de Durango, México. Ecosistemas y Recursos Agropecuarios 8(3): e3049. DOI: 10.19136/era.a8n3.3049
RESUMEN. El estado de Durango posee condiciones ambientales ideales para incrementar el desarrollo acuícola, pero en los últimos años ha disminuido la producción de esta actividad y descendido a los últimos lugares a nivel nacional. Una de las alternativas para un desarrollo acuícola sustentable en el estado es la aplicación de la Tecnología Biofloc (BFT) en unidades de producción de tilapia. El objetivo del presente estudio fue determinar la aptitud territorial del estado para la producción de tilapia (Oreochromis niloticus) con BFT. Se realizó una evaluación multicriterio mediante suma ponderada con apoyo de un grupo de expertos. También se generó un mapa de aptitud para la actividad mediante Sistemas de Información Geográfica en el que se establecieron cinco categorías de aptitud: muy baja, baja, media, alta y muy alta. La superficie estimada con aptitud alta y muy alta fue de $50182 \mathrm{~km}^{2}$ para desarrollar la actividad en el estado, las regiones con mayor aptitud se encontraron en el centro este y norte de la entidad, principalmente en la zona semiárida. El $39.67 \%$ de la superficie con aptitud alta y muy alta estimada se concentró en los municipios con un Índice de Desarrollo Humano (IDH) Medio - Bajo, lo cual representa un área de oportunidad para incrementar la seguridad alimentaria en estos municipios. Los resultados ayudarán en la identificación de sitios aptos para la implementación de proyectos acuícolas en el estado con enfoque óptimo en el uso del recurso hídrico.

Palabras clave: Escasez de agua, Índice de Desarrollo Humano, ordenamiento acuícola, seguridad alimentaria, Sistemas de Información Geográfica.

ABSTRACT. Durango state has ideal environmental conditions to increase aquaculture development; however, in recent years the production of this activity has decreased and descended to the last places at the national level. One of the alternatives for a sustainable aquaculture development in the state is the application of Biofloc Technology (BFT) in production units of tilapia. The aim of this study was to determine the land suitability of the state for tilapia (Oreochromis niloticus) production with BFT. A multi-criteria evaluation was carried out by means of weighted sum with the support of a group of experts. An aptitude map for the activity was generated through Geographic Information System in which five categories of aptitude were established: very low, low, medium, high and very high. An area with high and very high aptitude of $50182 \mathrm{~km}^{2}$ was estimated to develop the activity in the state, the regions with the highest aptitude were found in the central east and north of the state, mainly in the semi-arid zone. $39.67 \%$ of the high and very high aptitude estimated for the activity was concentrated in the municipalities with a Medium or Low Human Development Index (HDI), which represents an opportunity to increase food security in these municipalities. The results of this study will allow identifying suitable sites for implementing aquaculture projects in the state with an optimal approach in the use of water resources.

Key words: Aquaculture management, food security, Geographic Information System, Human Development Index, water scarcity. 


\section{INTRODUCCIÓN}

La acuicultura es una de las actividades productivas con mayor desarrollo en el mundo, con una tasa promedio de crecimiento anual del $5.3 \%$ en las últimas dos décadas (FAO 2020). En México, la importancia de la actividad acuícola no es la excepción, en el 2017 se registró una producción total de 404 $000 \mathrm{t}$, con un valor aproximado de 17813 millones de pesos, siendo los estados de Sonora, Sinaloa, Jalisco y Veracruz los principales productores (CONAPESCA 2018). Pero la forma en que se lleva a cabo la mayor parte de la actividad acuícola en México es todavía poco tecnificada y deficiente en el uso de recursos (Platas y Vilaboa 2014). Por ejemplo, la mayoría de las 4623 unidades producción de tilapia en México son extensivas o semi-intensivas con un uso poco eficiente del recurso agua (Betanzo-Torres et al. 2019). En este contexto, la producción de algunas especies dulceacuícolas con tecnologías que optimizan el uso de los recursos, especialmente el agua, y que incrementan la producción por unidad de volumen, se convierten en una alternativa de diversificación productiva viable (Platas y Vilaboa 2014). Tal es el caso de la Tecnología Biofloc (BFT), la cual se considera de recambio cero; a diferencia de la producción convencional acuícola donde se tiene la necesidad de recambiar hasta un $100 \%$ del volumen de agua por día para mantener su calidad, con esta tecnología solo se repone un mínimo de volumen de agua, principalmente por pérdida por evaporación (Collazos-Lasso y Arias-Castellanos 2015). Bajo este sistema, el recambio mínimo de agua y la eventual acumulación de compuestos tóxicos para las especies, como el amonio, se compensa mediante la adición de una fuente de carbono al sistema, logrando un balance deseado en la relación carbono-nitrógeno $(\mathrm{C}: \mathrm{N})$ que va de $11: 1$ a 20:1, lo que promueve la actividad de cierto tipo de bacterias heterótrofas (Crab et al. 2012). Las especies con sistemas filtradores como la tilapia (Oreochromis niloticus) son ideales para desarrollo de cultivos con esta tecnología (Avnimelech 2009). Debido al uso eficiente del recurso agua (ahorro de más del 90\%) aunado a la capacidad de producción de calidad en superficies reducidas, ha- cen de la BFT un modelo con potencial para implementarse en zonas con limitada disponibilidad de agua, como el norte de México (Betanzo-Torres et al. 2019). A pesar de sus características geográficas, físicas, hidrológicas, de extensión y climáticas favorables para el desarrollo acuícola, el estado de Durango ha pasado en los últimos años (2011-2018) del lugar 19 al 26 en producción acuícola a nivel nacional (SIAP 2018). El uso del agua, sobre todo para la parte del semidesierto en el norte del estado, tiene poca diversificación productiva; los ciclos de apertura y cierre de compuertas en los dos grandes embalses de la región y la dinámica de las actividades de pozos profundos y estanques, está determinada principalmente para actividades agrícolas y ganaderas, en particular para producción de forrajes (Pedroza et al. 2014). Además, el uso casi único del recurso hídrico en la región aumenta la dependencia hacia ciertas actividades productivas que en su mayoría son poco eficientes en el uso del agua (Ramírez et al. 2019). Lo anterior, pone de manifiesto la necesidad de reorientar proyectos productivos con el objetivo de atender regiones marginadas de la entidad (Pradeepkiran 2019). En este sentido, en el estado de Durango se encuentran regiones con bajo Índice de Desarrollo Humano (IDH), lo que revela áreas de oportunidad para mejorar las condiciones de bienestar de las personas mediante políticas y acciones para el desarrollo, así como a través de la dotación focalizada de recursos públicos (CONABIO 2014). Por lo que se requiere incrementar el conocimiento acerca del potencial de estas regiones del estado para establecer proyectos productivos que contribuyan a la seguridad alimentaria y mejoren la calidad de vida de la población (Pradeepkiran 2019).

De acuerdo con Román (2014), las evaluaciones multicriterio son adecuadas para la planificación territorial, ya que permiten considerar diversas opiniones, establecer comparación entre diferentes escenarios y ponderaciones, mientras que los Sistemas de Información Geográfica (SIG) son una herramienta eficiente en la planeación y ordenamiento de la actividad acuícola en diferentes regiones del mundo (Ghobadi et al. 2021). Además estas herramientas han contribuido en la viabilidad de 
las unidades de cultivo al seleccionar sitios con mayor aptitud física para la actividad, lo que disminuye el conflicto con otras actividades productivas (Gimpel et al. 2018). Algunos estados del norte del país cuentan con Programas de Ordenamiento Acuícola, pero el estado de Durango carece de estudios sobre el tema, además de que no cuenta con estudios de zonificación o determinación de sitios potenciales para el establecimiento de esta actividad (BetanzoTorres et al. 2019, Betanzo-Torres et al. 2020). Por lo anterior, el objetivo del presente estudio fue determinar la aptitud territorial para desarrollar la producción de tilapia (O. niloticus) con la Tecnología Biofloc en el estado de Durango.

\section{MATERIALES Y MÉTODOS}

\section{Área de estudio}

El trabajo se realizó en el estado de Durango, el cual representa el cuarto lugar en extensión territorial en la República Mexicana con $123451 \mathrm{~km}^{2}$, lo que representa el $6.3 \%$ de la superficie nacional (Márquez-Linares 2017). En la entidad se reconocen cuatro eco-regiones generales: región de las Quebradas, región de la Sierra, región de los Valles y región Árida y Semiárida (González et al. 2017, INEGI 2017). Debido a su gran extensión y orografía contrastante, se presenta una amplia diversidad de tipos de climas. La temperatura promedio anual varía de $8{ }^{\circ} \mathrm{C}$ en regiones serranas, hasta $26{ }^{\circ} \mathrm{C}$ para la zona semiárida en el norte del estado, mientras que la precipitación total anual fluctúa de los $100 \mathrm{~mm}$ en zonas semiáridas hasta $1800 \mathrm{~mm}$ en las regiones de las quebradas en el sur y este de la entidad (Cortés 2017). El estado tiene una disponibilidad cercana a los $6393 \mathrm{hm}^{3}$ de agua por año, tiene al menos 21 ríos principales y ocho presas con capacidad de almacenamiento que va de los 45 a los $2873 \mathrm{hm}^{3}$ (CONAGUA 2009, INEGI 2017).

\section{Identificación de atributos ambientales y determi- nación de criterios}

El mapa de aptitud para el establecimiento de unidades de cultivo de tilapia con BFT se desarrolló mediante una evaluación multicriterio, utilizando el método de suma ponderada (Román 2014). El análisis se realizó mediante la estandarización de cada criterio, asignando un valor de importancia con respecto a los demás criterios y se evaluaron todos los criterios en conjunto. La identificación de los atributos ambientales, entendidos como las variables cuantitativas o cualitativas que favorecen el desarrollo de las actividades humanas, y la determinación de los criterios se llevó a cabo por medio de la consulta con expertos y la búsqueda en literatura especializada disponible sobre proyectos de producción de tilapia con BFT, potencial de zonas en México para establecimiento de proyectos con esta tecnología, análisis de casos de éxito en unidades de cultivo de tilapia y otras especies dulceacuícolas, considerando las variables físicas y de infraestructura urbana disponible en fuentes de información cartográfica a nivel nacional e internacional (FAO 2014, Platas y Vilaboas 2014, Collazos-Lasso y Arias-Castellanos 2015, Chibras 2015, BetanzoTorres et al. 2019, Ghobadi et al. 2021). Fue establecido un grupo de 15 expertos en diferentes áreas de producción acuícola, cultivo de tilapia y uso de BFT, los cuales correspondieron a siete profesores investigadores, tres asesores técnicos, tres empresarios acuícolas y dos trabajadores de gobierno (Castillo y Peña-Cortés 2012). Un aspecto importante en la elección del grupo de trabajo, fue la representatividad de los integrantes en los diferentes sectores involucrados en investigación y producción acuícola con BFT. Los integrantes del grupo de expertos a través de un formulario validaron los criterios previamente identificados y llevaron a cabo la ponderación de los mismos mediante una matriz de decisión donde todos los criterios se compararon entre sí para determinar si tenían una mayor, menor o igual importancia por medio de juicios de valor.

\section{Estandarización de criterios}

Debido a que los criterios seleccionados estaban expresados en diferentes unidades fue necesario llevar a cabo una estandarización por medio de la transformación lineal de escalas utilizando el procedimiento de rangos, como se expresa en la siguiente fórmula: 


$$
V i j=\frac{x_{\max }-x_{i j}}{x_{\max }-x_{\min }}
$$

Donde: $x_{\min }$ es el valor mínimo para el j-ésimo cri-

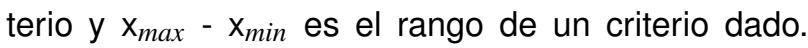
Aplicando la fórmula anterior, los valores normalizados para cada criterio oscilan entre 0 y 1 (Román 2014).

\section{Proyección espacial para la determinación de zonas con aptitud para producción de tilapia con BFT}

Los mapas de criterios se procesaron en el Sistema de Información Geográfica (QGIS 3.14) utilizando como fuentes de información el Instituto Nacional de Estadística y Geografía (INEGI 2013, INEGI 2014a, 2014b, INEGI 2019a, 2019b) y WorldClim 2 (Fick y Hijmans 2017), delimitando las capas bioclimáticas al tamaño del polígono del Estado de Durango y empleando un tamaño de celda de una hectárea. Para los criterios de cercanía a líneas eléctricas, cercanía a carreteras y caminos y cercanía a fuentes de agua superficial o subterránea se calculó la distancia euclidiana, mientras que los criterios de pendiente del terreno y temperatura media fueron remuestreados mediante el método de interpolación bilineal para homologar el tamaño de celda (SalasAguilar y Paz-Pellat 2018). Una vez que se contó con los mapas de los criterios estandarizados mediante la transformación lineal, se llevó a cabo la suma ponderada considerando los valores propuestos por el grupo de expertos. Los valores obtenidos en la suma ponderada fueron reclasificados por cuantiles en cinco categorías de acuerdo a su aptitud (muy baja, baja, media, alta y muy alta) y se determinó el porcentaje de cada categoría de aptitud a nivel estatal y municipal.

\section{Superposición de las capas de aptitud e Índice de Desarrollo Humano}

La capa de la aptitud reclasificada se sobrepuso con la capa del IDH a nivel municipal y se generó un mapa bivariable considerando las categorías de ambas capas (PNUD 2019). A partir de dicha superposición, se identificaron las regiones del estado de Durango con IDH Bajo y Medio y que contaban con una aptitud alta y muy alta para el cultivo de tilapia con BFT.

\section{RESULTADOS}

\section{Análisis multicriterio}

Se seleccionaron y priorizaron seis atributos ambientales para el desarrollo de unidades de producción de tilapia con BFT en el estado de Durango: energía eléctrica, disponibilidad de agua, temperatura, localidades, accesibilidad y pendiente del terreno. Los criterios con la ponderación mayor por parte del grupo de expertos, fueron la cercanía a las líneas eléctricas (29\%) debido a los requerimientos técnicos propios de la BFT, seguido de la cercanía a fuentes de agua superficial y subterránea (24\%). Los atributos ambientales, sus criterios y la ponderación obtenida se observan en el Tabla 1.

\section{Aptitud territorial para cultivo de tilapia con BFT}

Se establecieron cinco categorías de aptitud: muy baja, baja, media, alta y muy alta. El mapa general de aptitud, así como las superficies estimadas para cada una de las cinco categorías se observan en las Figuras 1 y 2 , respectivamente. EI $40.69 \%$ del territorio del estado de Durango se estimó con aptitud alta o muy alta para el establecimiento de unidades de cultivo de tilapia con BFT (Figura 3). Las áreas más extensas con estas categorías se determinaron para la parte centro este y noreste del estado, destacando amplias zonas de la región del semidesierto y particularmente de la Comarca Lagunera de Durango. Estas zonas estimadas con aptitud alta y muy alta para la actividad, destacan por presentar temperatura media anual cálida (mayor a 15 ${ }^{\circ} \mathrm{C}$ ), una red de infraestructura eléctrica bien establecida, disponibilidad de agua superficial y subterránea destinada principalmente para riego agrícola, cercanía a carreteras y localidades, además de que son sitios en su mayoría con una escasa pendiente del terreno. Las zonas con aptitud estimada como muy 
Tabla 1. Atributos ambientales, criterios y ponderación por el grupo de expertos.

\begin{tabular}{ccc}
\hline Atributo & Criterio & Ponderación (\%) \\
\hline Energía eléctrica & Cercanía a líneas eléctricas & 29 \\
Disponibilidad de agua & Cercanía a fuentes de agua superficial o subterránea & 24 \\
Temperatura & Temperatura promedio anual cálida & 19 \\
Localidades & Cercanía a localidades & 14 \\
Accesibilidad & Cercanía a carreteras y caminos & 9 \\
Pendiente del terreno & Pendiente plana o casi plana & 5 \\
\hline
\end{tabular}

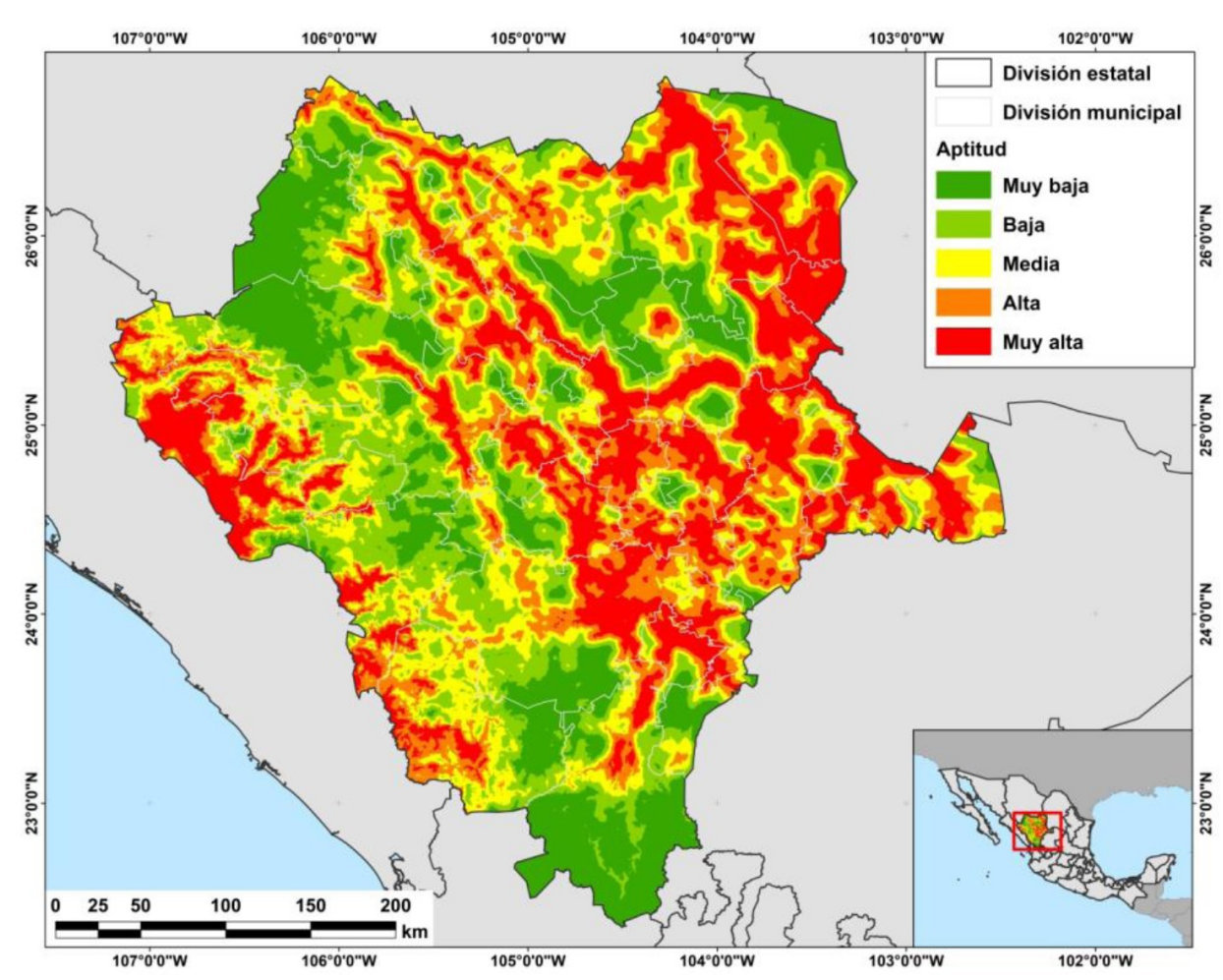

Figura 1. Mapa de aptitud para cultivo de tilapia con BFT en el estado de Durango.

baja, baja o media presentan distribución principalmente en las zonas de la Sierra Madre Occidental, donde si bien, existe mayor disponibilidad del recurso agua, tiene limitada la red eléctrica, además de temperaturas medias anuales bajas. De igual manera en extensas zonas del semidesierto en el norte del estado se presentan temperaturas medias cálidas, pero el factor imitante para la actividad es la disponibilidad de fuentes de agua superficial o subterránea.

\section{Aptitud territorial de acuerdo al IDH de los munici- pios}

En el estado existen 19 municipios con un IDH en categoría media y un municipio (Mezquital) en categoría baja (IDH < 0.55). Un área total de $19909.10 \mathrm{~km}^{2}$ con aptitud alta y muy alta fue estimada para estos municipios. Los municipios en estas categorías (IDH medio y bajo) que aportan mayor superficie de aptitud alta y muy alta para la actividad son General Simón Bolívar, Hidalgo, Indé, Mezquital, Pueblo Nuevo, San Dimas, San Juan de Guadalupe, San Juan del Río y Tamazula, para los cuales se estimaron $14755.4 \mathrm{~km}^{2}(74.11 \%)$ del total estimado para los municipios en esta condición. La representación cartográfica y los valores de superficie por municipio en esta categoría se observan en la Figura 4 y la Tabla 2. 


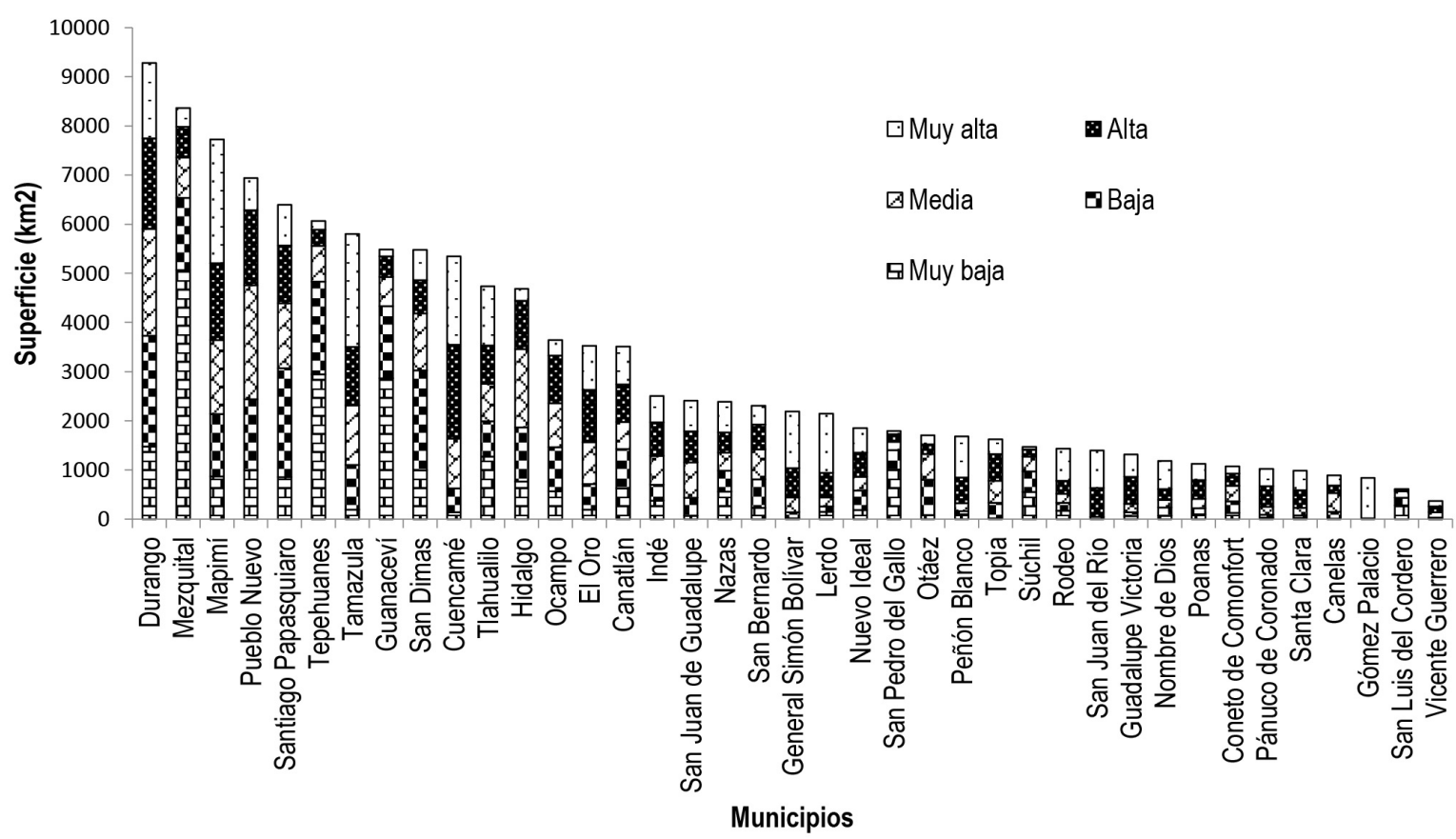

Figura 2. Áreas estimadas con diferente categoría de aptitud por municipio para el estado de Durango.

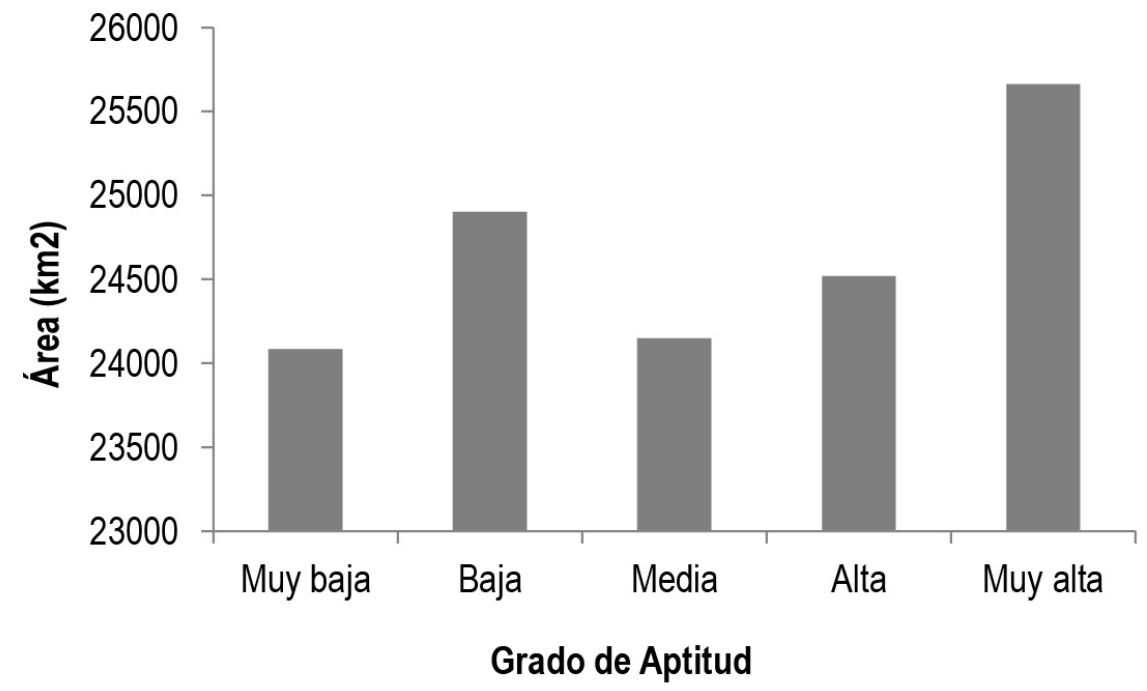

Figura 3. Áreas estimadas para diferentes categorías de aptitud para desarrollo de cultivo de tilapia con Tecnología Biofloc en el estado de Durango.

\section{DISCUSIÓN}

\section{Aptitud territorial para cultivo de tilapia con BFT}

La región del centro este y noreste del estado fue la zona con mayor aptitud para el cultivo de tila- pia con BFT, debido a que tiene una red de infraestructura eléctrica bien establecida, disponibilidad de agua superficial, suministrada principalmente por el Río Nazas, desde los municipios de San Bernardo, El Oro, Indé, pasando por Rodeo, Nazas, hasta la 


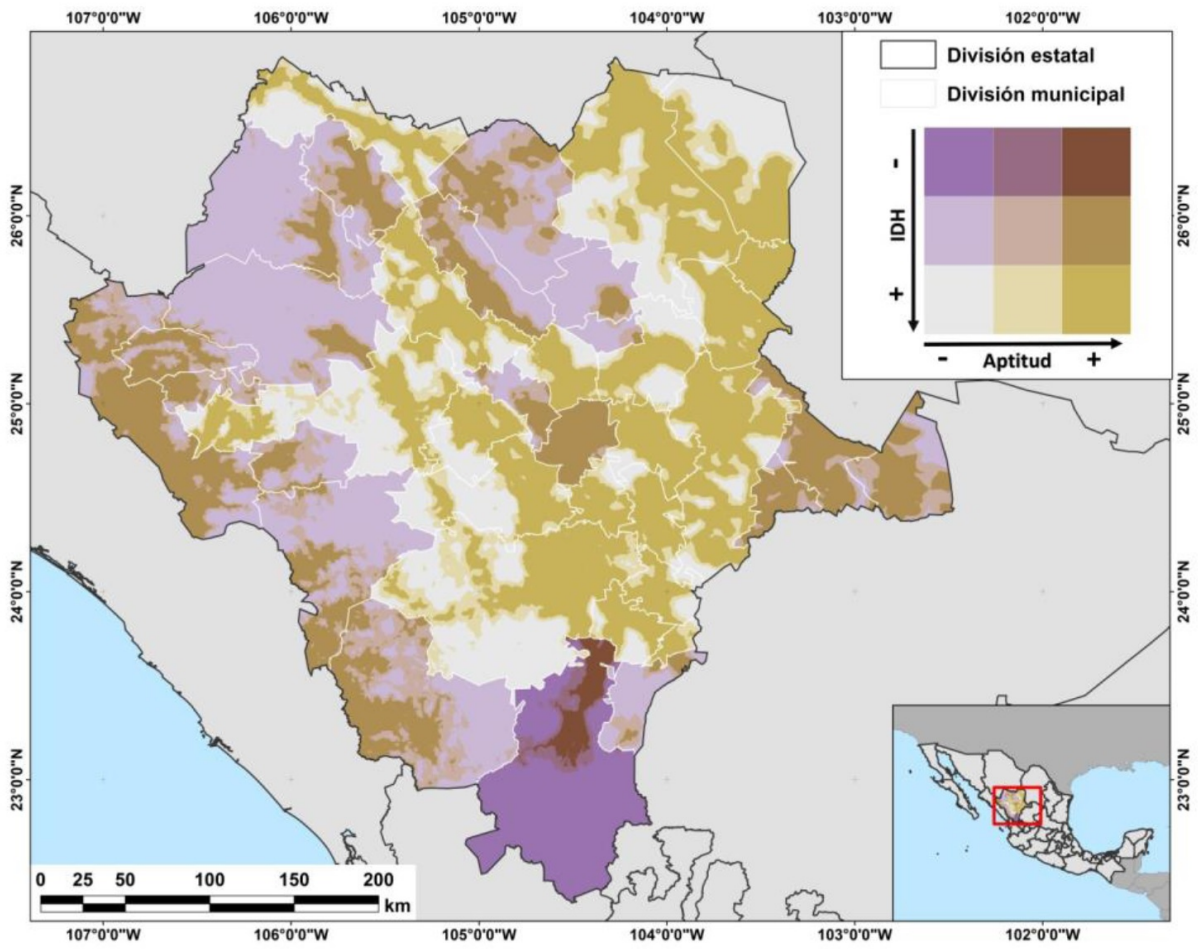

Figura 4. Aptitud alta y muy alta para la actividad, en zonas del estado con un íDH bajo y muy bajo.

Tabla 2. Municipios del estado con IDH Medio y Bajo y una aptitud alta y muy alta para la actividad.

\begin{tabular}{lccc}
\hline \multirow{2}{*}{ Municipio } & \multicolumn{2}{c}{ IDH } & \multirow{2}{*}{$\begin{array}{c}\text { Superficie con aptitud } \\
\text { alta y }\end{array}$} \\
\cline { 2 - 3 } & Valor & Categoría & alta $\left(\mathrm{km}^{2}\right)$ \\
\hline Canelas & 0.582 & Medio & 364.4 \\
Coneto de Comonfort & 0.649 & Medio & 396.1 \\
General Simón Bolívar & 0.65 & Medio & 1743.1 \\
Guanaceví & 0.639 & Medio & 555.7 \\
Hidalgo & 0.694 & Medio & 1226.6 \\
Indé & 0.665 & Medio & 1213.1 \\
Mezquital & 0.541 & Bajo & 1007.3 \\
Otáez & 0.588 & Medio & 386.5 \\
Pueblo Nuevo & 0.698 & Medio & 2179.4 \\
San Bernardo & 0.637 & Medio & 886.1 \\
San Dimas & 0.671 & Medio & 1293.3 \\
San Juan de Guadalupe & 0.65 & Medio & 1257.1 \\
San Juan del Río & 0.696 & Medio & 1351.8 \\
San Luis del Cordero & 0.676 & Medio & 36.8 \\
San Pedro del Gallo & 0.664 & Medio & 222.2 \\
Santa Clara & 0.684 & Medio & 757.7 \\
Súchil & 0.689 & Medio & 198.0 \\
Tamazula & 0.607 & Medio & 3483.6 \\
Tepehuanes & 0.671 & Medio & 505.3 \\
Topia & 0.624 & Medio & 844.9 \\
\hline
\end{tabular}

parte baja de la Cuenca en la Zona Metropolitana de la Laguna en los municipios de Lerdo y Gómez Palacio, y agua subterránea por la presencia de pozos para extracción de agua con fines agrícolas (BetanzoTorres et al. 2019). Además, la zona cuenta con promedios anuales de temperatura ambiental que os- 
cilan entre los 18 y $24{ }^{\circ} \mathrm{C}$, lo cual aumenta el potencial para el establecimiento de unidades de producción de tilapia con esta tecnología (Hernández et al. 2019). Al respecto, Betanzo-Torres et al. (2019) han descrito el potencial en el centro norte de México para el crecimiento acuícola con BFT, debido en parte a las condiciones antes descritas. Existe un contraste en la aptitud estimada para la actividad en los diferentes municipios del estado, debido a las características particulares de cada región. Los municipios que presentan una aptitud alta y muy alta en más del $70 \%$ de su superficie son Cuencamé, General Simón Bolívar, Gómez Palacio, Guadalupe Victoria, Lerdo, Pánuco de Coronado, Peñón Blanco, San Juan del Río y Santa Clara. Mientras que los municipios de Gómez Palacio y San Juan del Río tienen aptitud alta, en los cuales prácticamente el $100 \%$ de su superficie tiene aptitud alta y muy alta. El hecho de que extensas zonas estimadas con aptitud alta para el cultivo de tilapia con BFT se ubican en zonas metropolitanas o peri-urbanas (por ejemplo la Zona Metropolitana de la Laguna de Durango), lejos de ser una limitante para la actividad, es una oportunidad para el establecimiento de las unidades acuícolas con esta tecnología. Entre otras ventajas, la BFT tiene capacidad de alta producción en superficies reducidas (producción super-intensiva), a diferencia de la producción convencional, la cual requiere grandes extensiones de terreno para el desarrollo de la actividad, por la baja productividad de los sistemas (Crab et al. 2012). El desarrollo de acuicultura en ciudades para aumentar la seguridad alimentaria e incrementar la sustentabilidad ambiental es un fenómeno cada vez más documentado (Brooks y Conkle 2019, Mazumder y Datta 2019). Mientras que los municipios con menor superficie con aptitud en categoría alta y muy alta fueron Guanaceví, Hidalgo, Mezquital, Otáez, San Dimas, San Luis del Cordero, San Pedro del Gallo, Súchil y Tepehuanes. Estos municipios con baja aptitud se localizan en su mayoría en la Sierra Madre Occidental o su zona de influencia, donde a pesar de contar con una disponibilidad de agua relativamente alta, se presentan temperaturas promedio anual templadas $\left(8-16{ }^{\circ} \mathrm{C}\right)$, o en zonas del semidesierto con poca conectividad (incipiente red eléctrica) o escasez de fuentes de agua con fines agrícolas (Cortés 2017).

\section{Aptitud territorial de acuerdo al IHD de los munici- pios}

De acuerdo con PNUD (2019) el $51 \%$ de los municipios del estado tienen un IDH categorizado como Medio o Bajo, lo cual representa que sus habitantes carecen de algunas capacidades y libertades para tener una calidad de vida aceptable. La aptitud alta y muy alta estimada en estos municipios representa un área de oportunidad para la implementación de programas gubernamentales enfocados a la producción de tilapia con fines de autoconsumo o comercial con el enfoque de la BFT, debido a los múltiples beneficios. Mención especial merece el municipio de Mezquital en la zona sur de la entidad, el cual es el único municipio del estado con un IDH en categoría baja (0.54), lo cual implica un rezago en términos de ingreso per cápita, educación y calidad de vida (PNUD 2019). Por medio del presente estudio se estimó una superficie con aptitud alta y muy alta de $1007.3 \mathrm{~km}^{2}$ para la producción de tilapia con BFT en ese municipio, lo que representa una gran oportunidad de crecimiento productivo para contribuir a la seguridad alimentaria en el municipio. Cobra relevancia el hecho de que la producción acuícola bajo BFT demanda un alto respaldo de energía eléctrica, debido a la necesidad de mantener en constante movimiento y oxigenación el agua, lo cual promueve la formación de agregados o flóculos los cuales a su vez albergan diferentes organismos, principalmente bacterias heterotróficas que realizan la función de transformar compuestos nitrogenados potencialmente tóxicos en compuestos no tóxicos para los peces (Hernández et al. 2019). La importancia de este atributo (electricidad) se ve reflejada en la ponderación realizada por el grupo de expertos, al ser la variable más importante a tomar en cuenta para la aptitud de la actividad (Tabla 1). Lo anterior es trascendente debido a que a diferencia de otros sistemas de cultivo tradicionales, los realizados bajo la BFT pueden colapsar en cuestión de minutos por fallas eléctricas en el sistema, debido a la alta demanda de oxigenación (Choo y Caipang 2015, Tierney y Ray 2018, Betazo-Torres et al. 2020). 
Por otra parte se ha descrito un gran número de beneficios de la BFT en cultivos de tilapia, entre los que se encuentran la reducción significativa en el uso del recurso agua, producción de alimento natural para los peces en el mismo sistema, mejor factor de condición y calidad del producto final, supresión de enfermedades por presencia de microorganismos benéficos, incremento y mantenimiento de la calidad de agua, entre otros aspectos (Chaverra et al. 2017, Liu et al. 2019, Betanzo-Torres 2020). Por estos y otros beneficios, es altamente recomendable incrementar políticas públicas y mecanismos que incrementen la conversión de unidades de producción acuícola ya existentes e instalación de nuevas, en regiones con aptitud alta y muy alta del estado de Durango, con un enfoque de sustentabilidad en el uso de los recursos naturales. Con relación al beneficio de optimización del recurso agua, actualmente existe una crisis por escasez y mala calidad del recurso en el norte centro de México, problema que se acentúa en la zona semiárida del estado de Durango, principalmente la Comarca Lagunera. Además existen escenarios climáticos futuros en los cuales se prevé una disminución de la precipitación y aumento de la temperatura promedio para las próximas décadas para el norte de México, con lo cual se estima una menor disponibilidad del recurso agua para las diferentes actividades productivas y urbanas (Magaña et al. 2012, Martínez-Austria y Patiño-Gómez 2012). El uso de la BFT en unidades de producción actuales y futuras podría representar por una parte, una mayor diversificación en el uso productivo del agua, y por otra, una optimización en su uso con fines de incrementar la producción de alimento de buena calidad para autoconsumo o comercialmente y de esta forma contribuir a la seguridad alimentaria del estado.

A pesar de las ventajas como el ahorro significativo en el uso del recurso agua con la implementación de la BFT para la producción acuícola, existen limitantes que han impedido que esta tecnología se implemente en diferentes regiones de México. Estas limitaciones van desde el alto conocimiento técnico necesario para implementar la tecnología, escasa capacitación del personal encargado de las unidades de producción, limitada capaci- dad de infraestructura, unidades productivas diversificadas en actividades e incluso desconocimiento y desinterés por los beneficios de la tecnología (Betanzo-Torres et al. 2020). De esa manera se pone de manifiesto la importancia de incrementar el conocimiento científico de las condiciones propias de cada región con aptitud para implementación de esta actividad, así como el evaluar a escala comercial la aplicación de la BFT, debido a que la mayoría de los trabajos reportados para México son solo a nivel de investigación en centros especializados (BetanzoTorres et al. 2019). Está documentado que la estructura de las comunidades bacterianas que son básicamente el motor de los sistemas biofloc, son diferentes de una región a otra por las condiciones climáticas, físicas y ecológicas de las diferentes regiones, lo cual pone de manifiesto la necesidad de evaluar la viabilidad y funcionamiento de unidades de producción acuícola bajo condiciones específicas de cada región (Cardona et al. 2016, Liu et al. 2019). Con la generación del mapa de aptitud para la implementación de producción de tilapia con BFT, se identificaron regiones prioritarias del estado con potencial acuícola con un enfoque de optimización del recurso agua. Esta información puede ser utilizada en instrumentos de planeación como ordenamientos acuícolas estatales, programas de ordenamiento ecológico y territorial, planes de desarrollo municipales y estatales, programas de manejo de Áreas Naturales Protegidas, entre otros (Alfaro y Peña-Cortés 2012, FAO 2018). La determinación de sitios con potencial para establecimiento de unidades de producción de tilapia es una determinación general, que se debe complementar con evaluaciones posteriores específicas para cada región del estado, como un análisis in situ para analizar la calidad del agua disponible, aspectos legales de tenencia de la tierra y restricciones en los programas de manejo de Áreas Naturales Protegidas (Ghobadi et al. 2021). En el mismo sentido, la categorización en algunas zonas del estado con una aptitud para la actividad media, baja o muy baja, no implica una exclusión total del cultivo de tilapia con BFT, pero el costo de inversión o conversión de unidades de producción será mayor que en zonas con categoría alta o muy alta, ya sea para incre- 
mentar y mantener la temperatura del agua en una zona fría a través de instalación de invernaderos $u$ otros mecanismos o para compensar la infraestructura eléctrica en una región aislada. Por el contrario, en zonas con aptitud alta y muy alta se conjuntan los factores ideales para la implementación de este tipo de tecnología, disminuyendo los riesgos y costos de inversión en comparación con zonas con baja aptitud (Betanzo-Torres 2019). Es importante precisar que si bien se estima una superficie considerable con aptitud alta y muy alta para la producción de tilapia con BFT en el estado de Durango, para futuras investigaciones es necesario complementar evaluaciones de aptitud para otros tipos de producción acuícola, incluyendo la producción con otras especies comerciales, potencial de producción de especies nativas, unidades mixtas o con cierto porcentaje de adopción de la BFT, sistemas de acuaponía, así como la evaluación de escenarios futuros de cambio climático para esta actividad productiva en el estado. Lo anterior, con la finalidad de impulsar la producción acuícola en la entidad con un enfoque de planeación estratégica y optimización de los recursos naturales.

\section{CONCLUSIONES}

Las regiones con mayor aptitud para la producción de tilapia con BFT fueron el centro este y el noreste del estado de Durango, en donde destacan los municipios de General Simón Bolívar, Gómez Palacio, Guadalupe Victoria, Lerdo, Pánuco de Coronado, Peñón Blanco, San Juan del Río y Santa Clara como los que presentan la mayor superficie apta en proporción a su superficie total. Del total de la superficie con categoría de aptitud alta y muy alta estimada para el estado, el $39.67 \%$ corresponde para los municipios que tienen un IDH medio o bajo, lo cual representa un área de oportunidad para implementar proyectos acuícolas con un enfoque de uso óptimo del recurso agua para contribuir a la seguridad alimentaria del estado y mejorar la calidad de vida en regiones marginadas de la entidad.

\section{AGRADECIMIENTOS}

Al Programa para el Desarrollo Profesional Docente de Tipo Superior de la Secretaría de Educación Pública por el financiamiento para el desarrollo del presente Proyecto, a través del Proyecto para Fortalecimiento de Cuerpos Académicos 511-6/201915066.

\section{LITERATURA CITADA}

Alfaro D, Peña-Cortés F (2012) Potencial acuícola en áreas preandinas de la Región de La Araucanía: conflictos de uso con la actividad turística. Revista de Geografía Norte Grande 51: 137-157.

Avnimelech Y (2009) Biofloc Technology - A practical Guide Book. The World Aquaculture Society. Baton Rouge, Louisiana, United States.182p.

Betanzo-Torres EA, Marín-Muñiz JL, Piñar-Álvarez MA, Celdrán-Sabater D, Mata-Alejandro H (2019) Análisis de la aplicación de la tecnología biofloc en la producción de tilapia (Oreochromis niloticus) en regiones rurales de México. Revista Internacional de Desarrollo Regional Sustentable 4: 42-58.

Betazo-Torres EA, Piñar-Álvarez MA, Sandoval-Herazo LC, Molina-Navarro A, Rodríguez-Montoro I, GonzálezMoreno RH (2020) Factors that limit the adoption of biofloc technology in aquaculture production in Mexico. Water 12: 1-23.

Brooks BW, Conkle JL (2019) Commentary: Perspectives on aquaculture, urbanization and water quality. Comparative Biochemistry and Physiology, Part C 217: 1-4. DOI: 10.1016/j.cbpc.2018.11.014. 
Cardona E, Gueguen Y, Magré K, Lorgeoux B, Piquemal D, Pierrat F, Noguier F, Saulnier D (2016) Bacterial community characterization of water and intestine of the shrimp Litopenaeus stylirostris in a biofloc system. BMC Microbiology 16: 157. DOI: 10.1186/s12866-016-0770-z

Castillo DA, Peña-Cortés F (2012) Potencial acuícola en áreas preandinas de la Región de La Araucanía: conflictos de uso con la actividad turística. Revista de Geografía Norte Grande 51: 137-157.

Chaverra SC, García JJ, Pardo SC (2017) Biofloc effect on juvenils Cachama blanca Piaractus brachypomus growth parameters. CES Medicina Veterinaria y Zootecnia 12: 170-180.

Chibras DE (2015) Sustentabilidad de la acuicultura en México: perspectivas desde un caso de estudio en la Costa Chica de Oaxaca. Interdisciplina 3: 161-191.

Choo HX, Caipang CMA (2015) Biofloc Technology (BFT) and its application towards improved production in freshwater tilapia culture. AACL Bioflux 8: 362-366.

Collazos-Lasso LF, Arias-Castellanos JA (2015) Fundamentos de la tecnología biofloc (BFT). Una alternativa para la piscicultura en Colombia. Una revisión. Urinoquia 19: 77-86.

CONAPESCA (2018) Anuario Estadístico de Acuacultura y Pesca 2018. Comisión Nacional de Acuacultura y Pesca. SADER. México. 277p.

CONAGUA (2009) Programa hídrico visión 2030 del estado de Durango. Secretaría de Medio Ambiente y Recursos Naturales. México. 204p.

CONABIO (2014) Índice de Desarrollo Humano por municipio, 2010. Escala 1: 250 000. Comisión Nacional para el Conocimiento y Uso de la Biodiversidad. México. http://www.conabio.gob.mx/informacion/gis/ Fecha de consulta: 02 de marzo de 2021.

Cortés A (2017) Clima. En: Cruz A, Castaños E, Valero J, Daniela E (Eds). La biodiversidad en Durango, estudio de estado. CONABIO. México. pp: 31-45.

Crab R, Defoirdt T, Bossier P, Verstraete W (2012) Biofloc technology in aquaculture: Beneficial effects and future challenges. Aquaculture 356-357: 351-356.

FAO (2014) Ordenamiento territorial rural. Conceptos, métodos y experiencias. Organización de las Naciones Unidas para la Alimentación y la Agricultura. Universidad de Buenos Aires. Ministerio de Agricultura, Ganadería y Pesca. Buenos Aires, Argentina. 540p.

FAO (2018) Zonificación acuícola, selección de sitios y áreas de manejo bajo el enfoque ecosistémico a la acuicultura. Organización de las Naciones Unidas para la Alimentación y la Agricultura. Roma. 62p.

FAO (2020) El estado mundial de la pesca y la acuicultura 2020. La sostenibilidad en acción. Organización de las Naciones Unidas para la Alimentación y la Agricultura. Roma. 223p.

Fick SE Hijmans RJ (2017) Worldclim 2: New 1-km spatial resolution climate surfaces for global land areas. International Journal of Climatology 37: 4302-4315.

Ghobadi M, Nasri M, Ahmadipari M (2021) Land suitability assessment (LSA) for aquaculture site selection via an integrated GIS-DANP multi-criteria method; a case study of lorestan province, Iran. Aquaculture 530: 1-12. DOI: $10.1016 /$ j.aquaculture.2020.735776

Gimpel A, Stelzenmüller V, Töpsch S. Galparsoro I, Gubbins M, Miller D, Murillas A, Murray AG, Pinarbași K, Roca G, Watret R (2018) A GIS-based tool for an integrated assessment of spatial planning trade offs with aquaculture. Science of the Total Environment 627: 1644-1655. 
González MS, González M, Márquez MA (2017) Ecorregiones. En: Cruz A, Castaños E, Valero J, Daniela E (Eds). La biodiversidad en Durango. Estudio de Estado. CONABIO. México. pp: 59-62.

Hernández LE, Londoño JI, Hernández KA, Torres LC (2019) Los sistemas biofloc: una estrategia eficiente en la producción acuícola. CES Medicina Veterinaria y Zootecnia 14: 70-99.

INEGI (2013) Conjunto de datos vectoriales de información topográfica escala 1:50 000, Serie III. Instituto Nacional de Geografía y Estadística. México. https://www.inegi.org.mx/temas/topografia/ Fecha de consulta: 02 de marzo de 2021.

INEGI (2014a) Conjunto de datos vectoriales de la carta de Aguas subterráneas escala 1:250 000 Serie I. Instituto Nacional de Geografía y Estadística. México. https://datos.gob.mx/busca/dataset/conjunto-dedatos-vectoriales-de-la-carta-de-aguas-subterraneas-escala-1-250-000-serie-i Fecha de consulta: 02 de marzo de 2021.

INEGI (2014b) Red Nacional de caminos. Instituto Nacional de Geografía y Estadística. México. https://www.inegi. org.mx/temas/viascomunicacion/ Fecha de consulta: 02 de marzo de 2021.

INEGI (2017) Anuario estadístico y geográfico de Durango 2017. Gobierno del Estado de Durango/INEGI. https://datatur.sectur.gob.mx/ITxEF_Docs/DGO_ANUARIO_PDF.pdf. Fecha de consulta 06 de octubre de 2020.

INEGI (2019a) Conjunto de datos vectoriales de información topográfica escala 1:50 000 . Instituto Nacional de Geografía y Estadística. México. https://www.inegi.org.mx/temas/topografia/ Fecha de consulta: 02 de marzo de 2021.

INEGI (2019b) Localidades de la República Mexicana escala: 1:250 000. Instituto Nacional de Geografía y Estadística. México. http://www.conabio.gob.mx/informacion/gis/ Fecha de consulta: 02 de marzo de 2021.

Liu H, Li H, Wei H, Zhu X, Han D, Jin J, Yang Y, Xie S (2019) Biofloc formation improves water quality and fish yield in a freshwater pond aquaculture system. Aquaculture 506: 256-269.

Magaña V, Zermeño D Neri C (2012) Climate change scenarios and potential impacts on water availability in northern Mexico. Climate Reaearch 51: 171-184.

Márquez-Linares MA (2017) Resumen ejecutivo. Contexto físico. En: Cruz A, Castaños E, Valero J, Daniela E (Eds). La biodiversidad en Durango. Estudio de Estado. CONABIO. México. pp: 17-18.

Martínez-Austria PF, Patiño-Gómez C (2012) Efectos del cambio climático en la disponibilidad de agua en México. Tecnología y Ciencias del Agua 3: 5-20.

Mazumder S, Datta D (2019) Spatial Pattern of Urban and Peri-Urban Aquaculture in Kolkata Metropolitan Area, India. International Journal of Innovative Studies in Sociology and Humanities 4: 34-43.

Pradeepkiran JA (2019) Aquaculture role in global food security with nutritional value: a review. Translational Animal Science 3: 903-910.

Pedroza A, Ríos JL, Torres M, Cantú JE, Piceno C, Yáñez LG (2014) Eficiencia del agua de riego en la producción de maíz forrajero (Zea mays L.) y alfalfa (Medicago sativa): impacto social y económico. Terra Latinoamericana 32: 231-239.

Platas DE, Vilaboa J (2014) La acuacultura mexicana: potencialidad, retos y áreas de oportunidad. Revista Mexicana de Agronegocios 35: 1065-1071.

PNUD (2019) Informe de Desarrollo Humano Municipal 2010-2015. Transformando México desde lo local. Programa de las Naciones Unidas para el Desarrollo. México: Offset Santiago. Ciudad de México, México. $348 p$. 
QGIS Development Team (2019) QGIS Geographic Information System. Open Source Geospatial Foundation Project. https://qgis.org. Fecha de consulta: 03 de mayo de 2021.

Ramírez BA, González A, Valdivia R, Salas JM, García JA (2019) Tarifas eficientes para el agua de uso agrícola en la Comarca Lagunera. Revista Mexicana de Ciencias Agrícolas 10: 539-550.

Román ME (2014) Metodologías Multicriterio para el Ordenamiento Territorial. En: Paruelo JM, Jobbágy EG, Laterra P, Dieguez H, García MA, Panizza A (ed). Ordenamiento territorial rural. Conceptos, métodos y experiencias. Universidad de Buenos Aires. Ministerio de Agricultura, Ganadería y Pesca. Organización de las Naciones Unidas para la Alimentación y la Agricultura. Buenos Aires, Argentina. pp: 198-231.

SIAP (2018) Producción Pesquera por Estado: Durango. Servicio de Información Agroalimentaria y Pesquera: México. http://www.campomexicano.gob.mx/raw_pesca_gobmx/seccionar.php. Fecha de consulta: 15 October 2018.

Salas-Aguilar VM Paz-Pellat F (2018) Desarrollo de una base de datos climática nacional anual, 1989-2012: resolución $250 \mathrm{~m}$. Elementos para Políticas Públicas 2: 1-13.

Tierney TW, Ray AJ (2018) Comparing biofloc, clear-water, and hybrid nursery systems (Part I): Shrimp (Litopenaeus vannamei) production, water quality, and stable isotopedynamics. Aquacultural Engineering 82: 7379. 